\title{
Temporal Series Analyses of Cosmic-Ray-induced- neutron Spectra Measured in High-altitude During the Last Two Decades
}

\author{
HUBERT Guillaume ${ }^{1}$ \\ ONERA DPHY, University of Toulouse \\ 2 avenue Edouard Belin, 31055 Toulouse, France \\ E-mail: guillaume.hubert@onera.f \\ VERNETTO Silvia \\ Istituto Nazionale di Fisica Nucleare (INFN) and Osservatorio Astrofisico di Torino \\ Via P. Giuria 1, 10125, Torino, Italy \\ E-mail: vernetto@to.infn.it
}

\section{ZANINI Alba}

Istituto Nazionale di Fisica Nucleare (INFN)

Via P. Giuria 1, 10125, Torino, Italy

E-mail: zanini@to.infn.it

\begin{abstract}
During the last two decades, major efforts were conducted to characterize the Cosmic-Ray induced neutron spectra in high-altitude stations under different geomagnetic/altitude conditions. Analyses proposed in this work are based on neutron spectra measurements (four stations: Chacaltaya Cosmic Ray Laboratory, Testa Grigia Research Station, Pic du Midi observatory and Concordia polar station) and transport simulations based on modelling of atmospheric cascades according to primary spectra which only depend on the solar modulation potential. Analyses focused on count rates, fluxes and neutron spectra. Complementary calculations allowed to deduce the solar potential and to assess the dose level. Trends of solar potential values issued from the neutron spectra are quite consistent. The monitoring of the neutron fluxes and the neutron dose rate show seasonal oscillations in the Pic du Midi while the Concordia was not impacted. Analyses show the interest to monitor continuously the neutron spectra in distributed high-altitude stations.
\end{abstract}

36th International Cosmic Ray Conference -ICRC2019-

July 24th - August 1st, 2019

Madison, WI, U.S.A.

\section{$1 \quad$ Speaker}

(C) Copyright owned by the author(s) under the terms of the Creative Commons 


\section{Introduction}

The main secondary particles produced by the interaction of primary cosmic rays (CR) with the nuclei of the constituents of the atmosphere are neutrons, protons, muons, pions and electrons together with electromagnetic radiation [1]. The Neutron Monitors (NMs) worldwide distributed, with more than 50 neutron monitor stations, are used to measure and record the variability of cosmic ray flux, on the base of secondary neutron detection [2,3]. During the last two decades, major efforts were conducted to characterize the Cosmic-Ray (CR) induced neutron spectra in high-altitude stations under different geomagnetic/altitude conditions. Several spectrometric systems were operated in high-altitude stations to study neutron spectra and their dynamics. Instruments were mainly based on passive dosimetry systems [4] and Bonner sphere systems (BSS)[5]. Temporal series constitute an opportunity to investigate the time dependence of the CR induced neutron spectra with respect to the variation of the primary GCR correlated to solar activity, reaching the Earth magnetosphere.

This paper presents analyses of data recorded, including data obtained in the Chacaltaya Cosmic Ray Laboratory, the Testa Grigia Laboratory, the Pic du Midi observatory and the Concordia polar station. Experimental measurements are coupled to transport simulations based on atmospheric cascades modelling, assuming that primary CR spectra only depend on the solar modulation potential [6] the temporal variation of this parameter is investigated from coupled cascade neutron measurements.

\section{Experimental setup and modelling}

\subsection{Instrumentation}

Neutron spectral measurements have been performed with different spectrometric systems. In the Chacaltaya $\left(16^{\circ} 21^{\prime} \mathrm{S}, 68^{\circ} 7^{\prime} \mathrm{W}\right)$ site and in the Testa Grigia Research Station $\left(45^{\circ} 56^{\prime} \mathrm{N}\right.$, $\left.7^{\circ} 42^{\prime} \mathrm{E}\right)$, measurements are based on passive neutron detectors with different threshold and energy responses, allowing the reconstruction of the neutron spectra in the energy range of interest. A first system includes four passive detectors, i.e. bubble dosimeters BD100R (100 $\mathrm{keV}$ to $20 \mathrm{MeV}$ ), polycarbonate detector foils (1-150 MeV), polycarbonate detector bottles (1$150 \mathrm{MeV}$ ) and fission detectors based on 209Bi layers (100MeV to $400 \mathrm{GeV})[7,8]$. It allows to record spectra in the energy range from $100 \mathrm{keV}$ to $400 \mathrm{GeV}$. This system was calibrated at CERF (CERN EU High Energy Reference Field) [9]; which reproduces the neutron spectrum at flight altitudes and therefore is a suitable reference field for instrument calibration. A second system is based on the passive Bubble Detector Spectrometer (BDS) and concerns the energy range from $10 \mathrm{keV}$ to $20 \mathrm{MeV}$. It is composed by six different types of detectors, with different chemical composition implying different energy threshold $(10,100,600 \mathrm{keV}, 1,2.5$ and 10 $\mathrm{MeV}$ ). In both cases the neutron energy spectrum is obtained by unfolding the detectors readings by using the appropriate detector responses and the unfolding package BUNTO [10]

A Bonner multi-sphere neutron spectrometer extended to high neutron energies (from meV to several $\mathrm{GeV}$ ) was developed to measure the energy spectrum of the $\mathrm{CR}$ induced neutrons, considering the energy range from $\mathrm{meV}$ to $\mathrm{GeV}$ [11]. As detailed in previous works [5,6], this system was composed of spherical $3 \mathrm{He}$ proportional counters surrounded with spherical PEHD 
(high density polyethylene) moderators with different thicknesses. Additionally, the spectrometer includes two PEHD spheres with inner tungsten and lead shells in order to increase the response to neutrons above $20 \mathrm{MeV}$. Electronic part dedicated to drive each signal, was composed to of the pre-amplifier and the linear amplifier. The output pulse signals are treated by a multi-channel analyzer, translated as spectrum or counts. The total counts of each detector were obtained by summing the total counts over a given integration time. The response functions (deduced from GEANT4 [12] and/or MCNPx [13] calculations) were used to convert the measured counting rates to neutron energy spectrum. The CR neutron energy spectra can be obtained by unfolding, by means of a deconvolution method, the measured counting rates, considering the response functions and the initial neutron spectrum at sea level observed by Goldhagen et al.[14]. The unfolding package GRAVEL was used for the BSS system.

\subsection{High Altitude Stations}

Table I presents the high-altitude station/observatory characteristics, i.e. geophysical data, cut-off rigidities, atmospheric depths and operated periods. Data allow for investigating the long period dynamics to analyze the spectral variation of cosmic-ray-induced neutron and the effects of local and seasonal changes, as well as the short period dynamics during solar flare events.

Table I: High-altitude station description, including geophysical data, cut-off rigidity, atmospheric depth, neutron instrument used and measurement period operated dates.

\begin{tabular}{|c|c|c|c|c|c|}
\hline & $\begin{array}{l}\text { Chacaltaya, } \\
\text { Bolivia }\end{array}$ & $\begin{array}{l}\text { Plateau Rosa, } \\
\text { Italy }\end{array}$ & $\begin{array}{l}\text { Pic du Midi, } \\
\text { France }\end{array}$ & $\begin{array}{l}\text { Pico dos } \\
\text { Dias, Brazil }\end{array}$ & $\begin{array}{l}\text { Concordia } \\
\text { station, } \\
\text { Antarctica }\end{array}$ \\
\hline $\begin{array}{l}\text { Principal } \\
\text { investigator(s) }\end{array}$ & INFN & INFN & ONERA & $\begin{array}{l}\text { ONERA, } \\
\text { IEAv }\end{array}$ & ONERA \\
\hline Acronym & - & - & ACROPOL & - & CHINSTRAP \\
\hline Altitude & $+5220 \mathrm{~m}$ & $+3480 \mathrm{~m}$ & $+2885 \mathrm{~m}$ & $+1685 \mathrm{~m}$ & $+3233 \mathrm{~m}$ \\
\hline Latitude & $16^{\circ} 21^{\prime} \mathrm{S}$ & $45^{\circ} 56^{\prime} \mathrm{N}$ & $42^{\circ} 55^{\prime} \mathrm{N}$ & $22^{\circ} 32^{\prime} \mathrm{S}$ & $75^{\circ} 06^{\prime} \mathrm{S}$ \\
\hline Longitude & $68^{\circ} 7^{\prime} \mathrm{W}$ & $7^{\circ} 42^{\prime} \mathrm{E}$ & $0^{\circ} 08^{\prime} \mathrm{E}$ & $45^{\circ} 34^{\prime} \mathrm{W}$ & $123^{\circ} 19^{\prime} \mathrm{E}$ \\
\hline Cut-off rigidity & $13.1 \mathrm{GV}$ & $5.4 \mathrm{GV}$ & $5.6 \mathrm{GV}$ & $9 \mathrm{GV}$ & $<0.001 \mathrm{GV}$ \\
\hline $\begin{array}{l}\text { Atmospheric } \\
\text { Depth }\end{array}$ & $530 \mathrm{~g} / \mathrm{cm}^{2}$ & $672.5 \mathrm{~g} / \mathrm{cm}^{2}$ & $700.7 \mathrm{~g} / \mathrm{cm}^{2}$ & $774.7 \mathrm{~g} / \mathrm{cm}^{2}$ & $635 \mathrm{~g} / \mathrm{cm}^{2}$ \\
\hline $\begin{array}{l}\text { Neutron } \\
\text { instrument }\end{array}$ & Passive systems & Passive systems & BSS & BSS & BSS \\
\hline Operated date & $\begin{array}{l}\text { November - } \\
\text { December } 1997\end{array}$ & January 1998 & $\begin{array}{l}\text { Continuously since } \\
\text { May } 2011\end{array}$ & $\begin{array}{l}\text { 2015, from } \\
\text { Jan. to May }\end{array}$ & $\begin{array}{l}\text { Since Dec. } \\
2015\end{array}$ \\
\hline
\end{tabular}

Measurements of atmospheric neutron spectra in extended energy range (100 keV to 400 $\mathrm{GeV})$ and short energy range (10 keV-20 MeV) have been carried out from 1997 to 2007 in the Chacaltaya Cosmic Ray Laboratory and the Testa Grigia Laboratory. The Chacaltaya Cosmic Ray Laboratory is located near La Paz, Bolivia, at an elevation of $5220 \mathrm{~m}$ above sea level corresponding. Testa Grigia Laboratory is at an elevation of $3480 \mathrm{~m}$ above sea level in the Italian Alps. Since 2008, some scientific equipments were operated in at the Pic du Midi Station, including a BSS neutron spectrometer, detectors based on and semiconductor and scintillator detectors. Measurements and analyses of the spectral variations of CR induced neutrons were performed over a height year period. A second neutron spectrometer was exploited simultaneously from February to May 2015 at the summit of the Observatory of the Pico dos Dias (OPD, Brazil, 1864 m a.s.l.), located in the region of South Atlantic Magnetic 
Anomaly (SAMA). A BSS neutron spectrometer was also operated in the Concordia Antarctic station since December 2015 in the framework of the CHINSTRAP project support by IPEV (French Polar Institute). This station is located at Dome C on the Antarctic Plateau at 3233m above the sea level, where an almost zero rigidity cut-off $(\mathrm{R}<0.01 \mathrm{GV})$, i.e., no geomagnetic shielding, allows to extend the investigation to low energy primary CRs.

\subsection{Modeling approach}

Some methods were proposed to model the secondary atmospheric CRs, some of which are based on nuclear transport simulations. The ATMORAD approach combines the GEANT4 simulations of extensive Air Showers to primary GCR spectra defined by the Force-Field Approximation [15] and which only depends on the solar potential $\phi$. The secondary CR spectra can be determined as a function of the altitude, the longitude, the latitude and the sun activity through the solar potential. The modulation potential $\phi(t)$ is only a formal parameter whose physical interpretation is not straightforward, especially on short time scales and during periods of active Sun. Virtually, everything is somehow correlated to overall solar activity, and some parameters like $\phi(t)$ and sunspot number are particularly relevant at providing an overview. Thus, several strategies have been developed for the reconstruction of time series of the modulation potential $\phi(t)$. Among these methods, an atmospheric radiation model named ATMORAD [16] based on GEANT4 simulations and Force-Field Approximation, allows to evaluate the modulation potential.

\section{Analyses of CR induced-neutron Spectra during the Last Two Decades}

Fig. 1 presents the atmospheric neutron spectra in lethargic representation, obtained by BSS system for the Chacaltaya station (a), the Testa Grigia Laboratory (b), the Pic-du-Midi observatory (c) and the Concordia station (d).

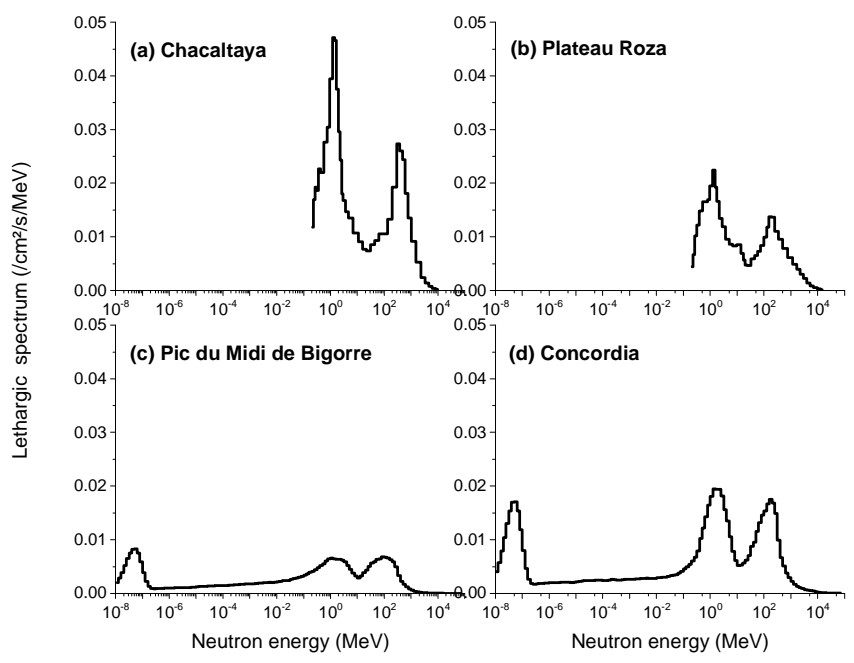

Fig. 1. Atmospheric neutron spectra in lethargic representation obtained in the Chacaltaya station (a), the Testa Grigia Laboratory (b), the Pic-du-Midi observatory (c) and the Concordia station (d). Concerning the Pic du Midi and Concordia, integration periods are considered from December 2015 to May 2019. 
Concerning the Pic du Midi and Concordia, integration periods extend from December 2015 to May 2019. It is clearly visible that the shapes of the spectra are very similar. Indeed, a maxwellian thermal peak below $0.5 \mathrm{eV}$ is observed for the Pic du Midi and Concordia data, an epithermal plateau between $0.5 \mathrm{eV}$ and $0.1 \mathrm{MeV}$, an evaporation peak from $0.1 \mathrm{MeV}$ to $20 \mathrm{MeV}$ and a cascade peak above $20 \mathrm{MeV}$. The evaporation and cascades peaks are observed for the two four locations. This part deals with the comparison of the energy distribution of the atmospheric neutrons according to the altitude.

The values of the various neutron fluence rates integrated over the energy domains D previously defined (i.e. thermal: $\dot{\varphi}_{t h}$, epithermal: $\dot{\varphi}_{\text {epi }}$, evaporation: $\dot{\varphi}_{\text {eva }}$, cascade: $\dot{\varphi}_{c a s}$ and total: $\dot{\varphi}_{\text {tot }}$ ) are reported on the Table II for the five stations.

Table II: neutron fluence rates integrated, in neutron $\mathrm{cm}^{-2} \cdot \mathrm{s}^{-1}$, over the thermal, epithermal, evaporation and cascade energy domains, for the five station presented in table I.

\begin{tabular}{|c|c|c|c|c|c|}
\hline & $\dot{\varphi}_{t h}$ & $\dot{\varphi}_{e p i}$ & $\dot{\varphi}_{\text {eva }}$ & $\dot{\varphi}_{\text {cas }}$ & $\dot{\varphi}_{t o t}$ \\
\hline Chacaltaya station & - & - & 0.097 & 0.067 & - \\
\hline Testa Grigia Lab. & - & - & 0.057 & 0.041 & - \\
\hline Pic du Midi & 0.016 & 0.02 & 0.026 & 0.020 & 0.081 \\
\hline Pico dos Dias & & & & & \\
\hline Concordia & 0.032 & 0.031 & 0.059 & 0.045 & 0.017 \\
\hline
\end{tabular}

Fig. 2(a) presents the temporal series of neutron fluxes derived from measurements in the Pic du Midi observatory and the Concordia station during the period from May 2011. Contributions induced by cascade neutrons are extracted and presented in Fig 2(b).

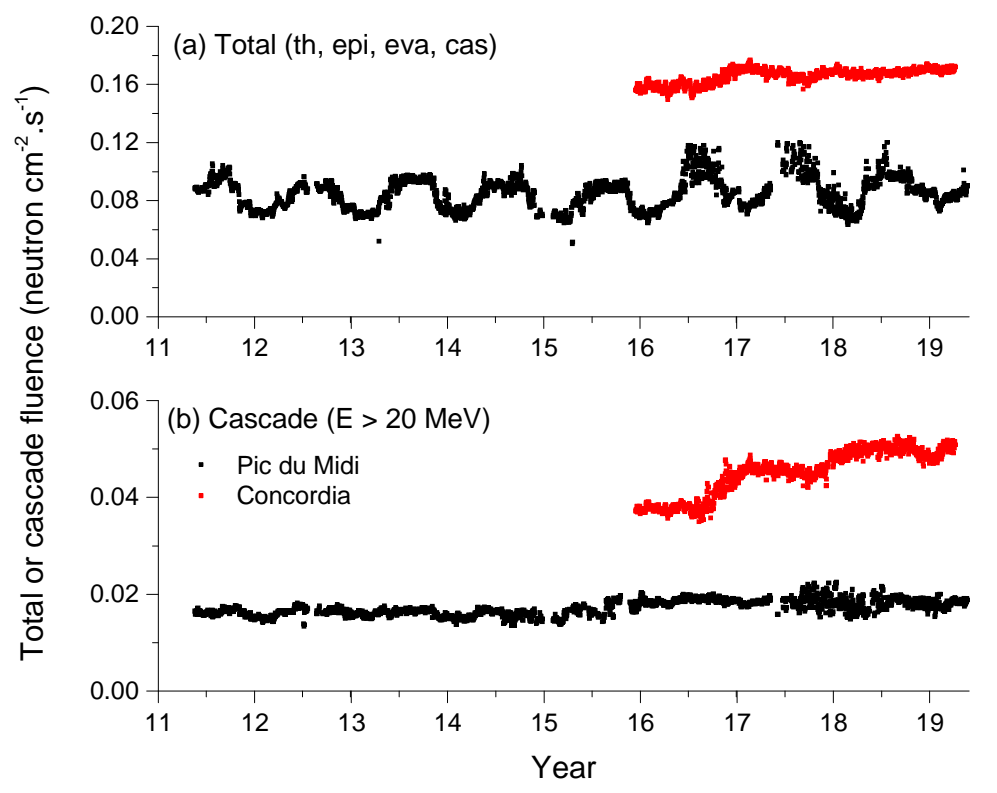

Fig. 2: Atmospheric neutron spectrum in lethargic representation obtained in the Pic-du-Midi observatory and the Concordia station during the period from December 2015 to May 2017.

Concerning the Pic-du-Midi measurements, a significant temporal oscillation is observed, mainly induced by snow thickness during winter. As shown in previous works $[15,16]$ seasonal 
oscillations are governed by ground albedo neutron impacts on thermal, epithermal and evaporation domains. Conversely, no oscillation is observed for Concordia measurements, due to the stability of the meteorological environment (i.e. a few centimeters of snow per year).

The increase in total flux measured in Concordia reflects the transition from strong to low solar activity. Indeed, the current solar cycle $\left(24^{\text {th }}\right)$ began in December 2008 and activity was minimal until early 2010. Cycle 24 reached its maximum in April 2014. Reversed polarity polar active sunspot regions observed from December 2016 indicate that the transitional phase to solar cycle 25 is in process. Moreover, cascade neutrons recorded in the Pic du Midi, because they are undisturbed by albedo, illustrate the dynamics of the solar cycle 24 .

\section{Analyses based on solar modulation analyses}

The solar potential $\varphi(t)$ is directly linked to the Sun's Activity. As showed in Fig. 2(b), cascade neutron fluxes were lowly impacted by the hydrogen density variations, allowing their uses to deduce the solar potential $\varphi(t)$. Fig. 3 shows the solar potential dynamics deduced from measurements operated in the four stations (Chacaltaya, Plateau Rosa, Pic du Midi and Concordia). The solar potential temporal series issued from Oulu were added [17] Trends issued from the Pic du Midi and Concordia are quite comparable with that referring to Oulu, with better results for Concordia due to the high level of detection (altitude and latitude).

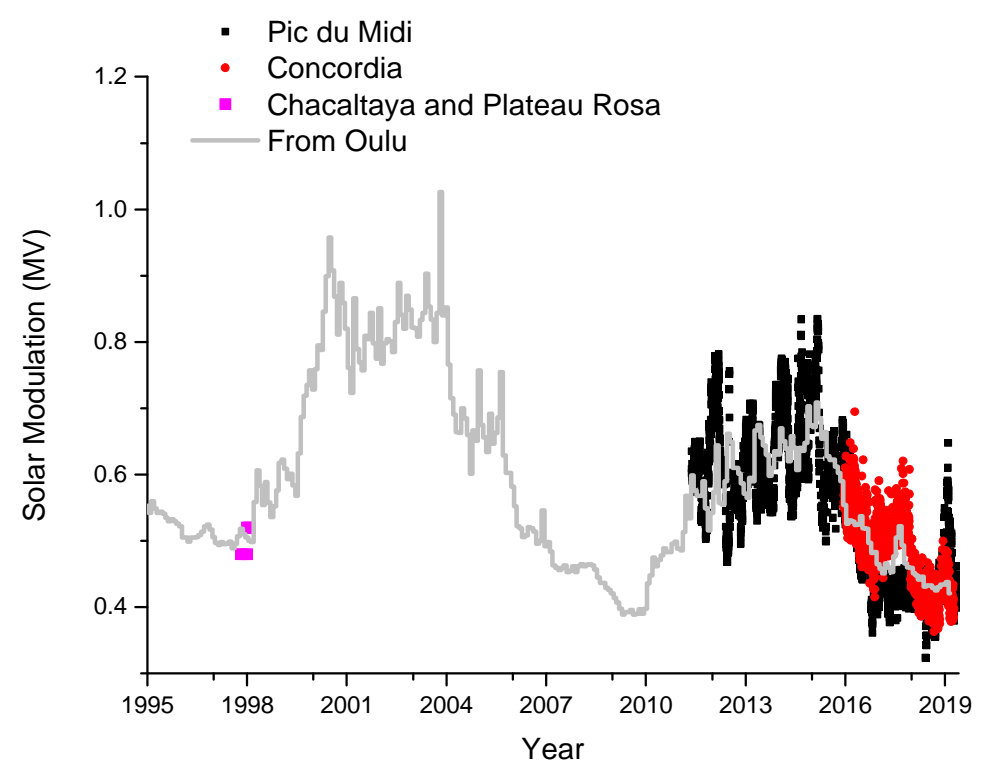

Fig. 3: Solar modulation in GV and from 2011, issued from cascade neutron flux measured in the Pic du Midi, the Concordia station, Chacaltaya and Plateau Rosa. Values issued from Oulu [17] are added.

\section{Neutron dose rate measurement}

Neutron spectra measurements over long time periods are also helpful for dosimetry studies. The monitoring of the neutron dose rate in high altitude and latitude sites is of great importance, since in these locations the flux of secondary cosmic ray is higher and more affected by the solar activity, with possible significant increases during very energetic solar events. To obtain the value of the neutron ambient dose equivalent from the neutron spectra, we 
used the flux-to-dose conversion factors given in [18] and [19]. Fig. 4 shows the ambient dose equivalent rate as a function of time, obtained from a set of neutron spectra measured at the Pic du Midi Observatory during 9 years and at the Concordia station during 3 years. The upper panel shows the total dose obtained considering all energy neutrons. The average dose rate in Concordia $\left(138 \mathrm{nSv} \mathrm{h}{ }^{-1}\right)$ is 2.3 times higher than the average dose in Pic du Midi (59 $\left.\mathrm{nSv} \mathrm{h}{ }^{-1}\right)$. The pattern in time is similar to that shown in Fig. 2 for the neutron flux. The dose rate, in particular in Pic du Midi, shows large annual oscillation due to local seasonal effects, with amplitudes reaching $30 \%$ in certain periods. The dose rate shown in the lower panel, obtained considering only cascade neutrons $(\mathrm{E}>20 \mathrm{MeV})$, is $2.5-3$ times lower and has a more stable behavior, except for a significant increase of about $15 \%$ in Concordia during 3 years (due to the lowering of solar activity).
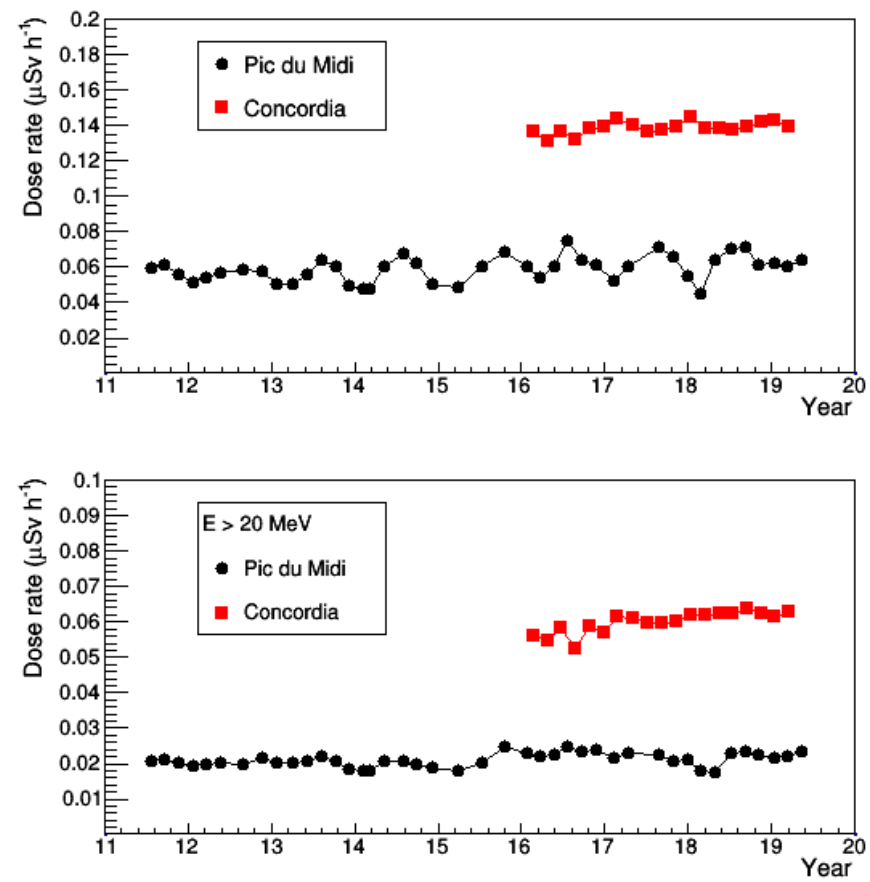

Fig.4. Neutron ambient dose equivalent rates vs. time for Pic du Midi Observatory and Concordia station. Upper panel: all neutrons. Lower panel: neutrons with energy larger than $20 \mathrm{MeV}$.

\section{Conclusion}

Measurements of the energy spectrum of CR recorded on high altitude stations during the last two decades are presented. Analyses concern data recorded, including data obtained in the Chacaltaya Cosmic Ray Laboratory, the Testa Grigia Laboratory, the Pic du Midi observatory and the Concordia polar station. Spectral measurements were performed by using passive neutron detectors with different threshold and energy responses in Chacaltaya and Testa Grigia laboratories. BSS spectrometers were used in Concordia and Pic du Midi observatories. Neutron fluence rates integrated over the four energy domains (i.e. thermal, epithermal, evaporation, and cascade) are also investigated for the four locations. The ratio between the cascade and epithermal domains is similar. 
In addition to the usual analyzes of count rates, fluxes or spectra, neutron spectra were used to investigate contributions induced by cascade neutrons and to deduce the solar potential. Trends issued from the neutron spectra are quite comparable with that referring to Oulu, with better results for Concordia due to the high level of detection (altitude and latitude). The last part is devoted to dosimetry studies. Indeed, the monitoring of the neutron dose rate in high altitude and latitude sites is of great importance, since in these locations the flux of secondary cosmic ray is higher and more affected by the solar activity, with possible significant increases during very energetic solar events. Seasonal oscillations due to environmental effects (humidity, snow thickness etc.) were observed. Analyses show the interest to monitor continuously the neutron spectra in distributed high-altitude stations.

\section{Acknowledgments}

The authors thank the technical staff for their supports at the Pic-du-Midi observatory and the technical staff of the Concordia station for their great help and hard work in harsh conditions.

\section{References}

[1] P. K. F. Grieder, 1st ed., pp. 1093, Elsevier, 2001.

[2] Neutron Monitor DataBase, available: http://www.nmdb.eu.

[3] J. Bieber et al., Journal of Geophysical Research: Space Physics, vol. 118, pp. 6847-6851, 2013.

[4] A. Zanini et al., Neutron spectrometry at various altitudes in atmosphere by passive detector techniques Nuovo Cimento. C; 24CS2, 691-697,2001.

[5] G. Hubert et al., Astroparticles Physics, vol. 74, pp. 27-36, 2016.

[6] G. Hubert et al., Radiation Research, vol. 184, issue 1, pp. 83-94, 2015.

[7] L.Tommasino et al., Cosmic-ray neutron spectrometry by solid state detectors. Radiation Measurements, vol. 36, pp. 307-311 2003

[8] L.Tommasino, Nuclear track detectors for charged particles and neutrons. International Conference for New Europe 2006, Portoroz, Slovenia, September 18-21, 2006.

[9] A. Ferrari, M. Silari, A., Mitaroff, A reference radiation facility for dosimetryat flight altitude and in space. Phys. Medica 17 (Suppl. 1), pp. 115-118, 2001.

[10] A. Zanini, et al., Neutron spectrometry at high mountain observatories. J. Atmos. Solar-Terrestrial Phys., vol. 67, pp. 755-762, 2005.

[11] A. Cheminet et al., IOP Science Journal of Instr., 2nd FNDA, vol. 7, 2012.

[12] S. Agostinelli et al., Geant4 - a simulation toolkit, Nuclear Instruments and Methods in Physics Research Section A: Accelerators, Spectrometers, Detectors and Associated Equipment, vol. 506, issue 3, pp. 250-303, 2003.

[13] A General Monte Carlo N-Particle (MCNP) Transport Code, https://mcnp.lanl.gov/

[14] P. Goldhagen, MRS Bulletin, vol. 28, issue 2, pp. 131, 2003.

[15] G. Hubert et al., Modeling of ground albedo neutrons to investigate seasonal cosmic ray-induced neutron variations measured at high-altitude stations, Journal of Geophysical Research: Space Physics, vol. 121, issue 12, pp. 12,186-12,201, 2016.

[16] G. Hubert, Ground Albedo Neutron impacts to Seasonal Variations of Cosmic-Ray-induced Neutron in medium Geomagnetic Latitude and Antarctica: Impacts on Soft Error Rate IEEE Transaction on Nuclear Science, vol. 64, issue 1, pp. 622-629, 2017.

[17] Oulu Cosmic Ray Station, https://cosmicrays.oulu.fi

[18] ICRP Publication 74. Ann. 219 ICRP 26 (3-4), 1996.

[19] M. Pelliccioni, Radiation Protection Dosimetry, vo. 88, issue 4, pp. 279-297, 2000. 